Revista Digital Universitaria

Vol. 22, Núm. 6, noviembre-diciembre 2021

\title{
Buscar el mejoramiento docente en la educación a distancia
}

\author{
Alexis Haro Piña
}

\begin{abstract}
Resumen
La educación siempre ha estado en el desarrollo del ser humano. Desde que los padres decidieron instruir a sus hijos en aspectos como la caza o el cuidado de sí mismos, ésta ha servido para mejorar la calidad de vida y, del mismo modo, ha evolucionado drásticamente con el paso del tiempo. En medio de la pandemia de la covid-19, los docentes nos hemos visto obligados a no tener sesiones educativas de forma presencial, a las cuales estábamos acostumbrados. Con ello, obtuvimos experiencias en las que se pone en evidencia que nos hace falta desarrollar habilidades para clases digitales. El objetivo principal de este artículo es mencionar las áreas de oportunidad que tenemos en la educación a distancia. Asimismo, se revisarán cuestiones como: ¿qué beneficios tiene la educación en línea?, ¿qué aspectos en contra tiene?, ¿qué problemáticas existen? y ¿qué áreas de oportunidad tenemos en este momento y cómo podemos implementarlas?
\end{abstract}

Palabras clave: apoyo, escuela secundaria, desempeño académico, participación de padres de familia, clases en línea.

\section{SEARCHING FOR TEACHER IMPROVEMENT IN ONLINE EDUCATION}

\begin{abstract}
Education has always been key for the development of human beings. Since parents decided to instruct their children in aspects such as hunting or taking care of themselves, it has served to improve the quality of life and, in the same way, it has drastically evolved over time. During coviD-19 pandemic, teachers have been forced not to have face-to-face educational sessions, to which we were accustomed. With this, we obtained experiences that evidence the need to develop skills in digital classes. The main objective of this article is to mention the areas of opportunity that we have in distance education. In the same way, we review questions such as: what benefits does online education have? What are its cons? What problems exist? What areas of opportunity do we have at this time and how can we implement them?
\end{abstract}

Keywords: support, online education, high school, academic performance, parent's involvement. 
Alexis Haro Piña

Profesor nivel secundaria en la Universidad Autónoma de la Laguna interesado en el mejoramiento de la educación. Es licenciado de educación secundaria con especialidad en Historia graduado de la Escuela Normal de la Laguna Cursos Regulares. En 2018 obtuvo el grado de Maestro en educación en la Universidad Autónoma de la Laguna. Posteriormente cursó el Doctorado en Desarrollo educativo en la Universidad Autónoma de la Laguna y concluyó este mismo en julio del 2021. Lleva ejerciendo la docencia por 7 años consecutivos.

\section{Introducción}

L

a finalidad de este artículo es discutir las áreas de oportunidad en la educación a distancia, así como las posibilidades de aprovechar este tipo de enseñanza al máximo. Lo anterior conllevará mejores resultados cuando tengamos la posibilidad de aplicar de nueva cuenta esta modalidad tanto a corto como a largo plazo. Los beneficiados en la lectura de este texto serán los docentes, instituciones, padres de familia y directivos. Con ello, se podrá generar una visión en las áreas de oportunidad que existen en la educación a distancia, para facilitar la mejora del nivel educativo en los nuevos ciclos.

Autores como Balarin, Tobon, Cabello y Borges nos hacen reflexionar que la educación a distancia tiene ya un tiempo con nosotros y que puede ser mejorada mediante el cambio en las áreas de oportunidad. Las ideas que estos autores nos otorgan hacen hincapié en acciones que aumentarán el nivel de efectividad en este tipo de enseñanza y, gracias a ello, podremos generar una educación que garantice eficiencia y no un estancamiento. Para concluir, tenemos la finalidad de determinar cómo mejorarla, así como poder sacarle provecho. 


\section{El contexto de la pandemia}

Debido a la aparición del virus SARS-CoV-2, la educación a nivel global tuvo que cambiar de forma radical durante 2020 y 2021. Aún con las vacunas disponibles al momento de escribir este artículo, esta pandemia estará con nosotros por más tiempo del que pensábamos y el volver a la normalidad es muy distante, por lo que se origina una problemática para el sistema educativo. Aunado a todo esto, los docentes hemos enfrentado dificultades en la modalidad de enseñanza a distancia, la cual no esperábamos y que nos tomó de sorpresa.

¿Qué problemáticas está enfrentando el sistema educativo? En el ámbito de las clases en línea, algunas de las principales dificultades son: el desconocimiento de las plataformas digitales, alumnos - junto a padres de familia- que no saben cómo se lleva a cabo la educación a distancia, docentes que palidecen ante la tecnología nueva, presión de organismos educativos que necesitan ofrecer el producto para poder existir e, incluso, abandono escolar. Estas son algunas de las problemáticas más notorias y que aún no hemos atendido a gran escala en México.

Si buscamos mejorar en este ámbito, debemos realizar cambios y modificaciones pertinentes entre los involucrados en el proceso de enseñanzaaprendizaje. No esperábamos que esta nueva modalidad de educación fuera tan determinante y el que estemos en medio de una pandemia nos ayuda a entender su importancia.

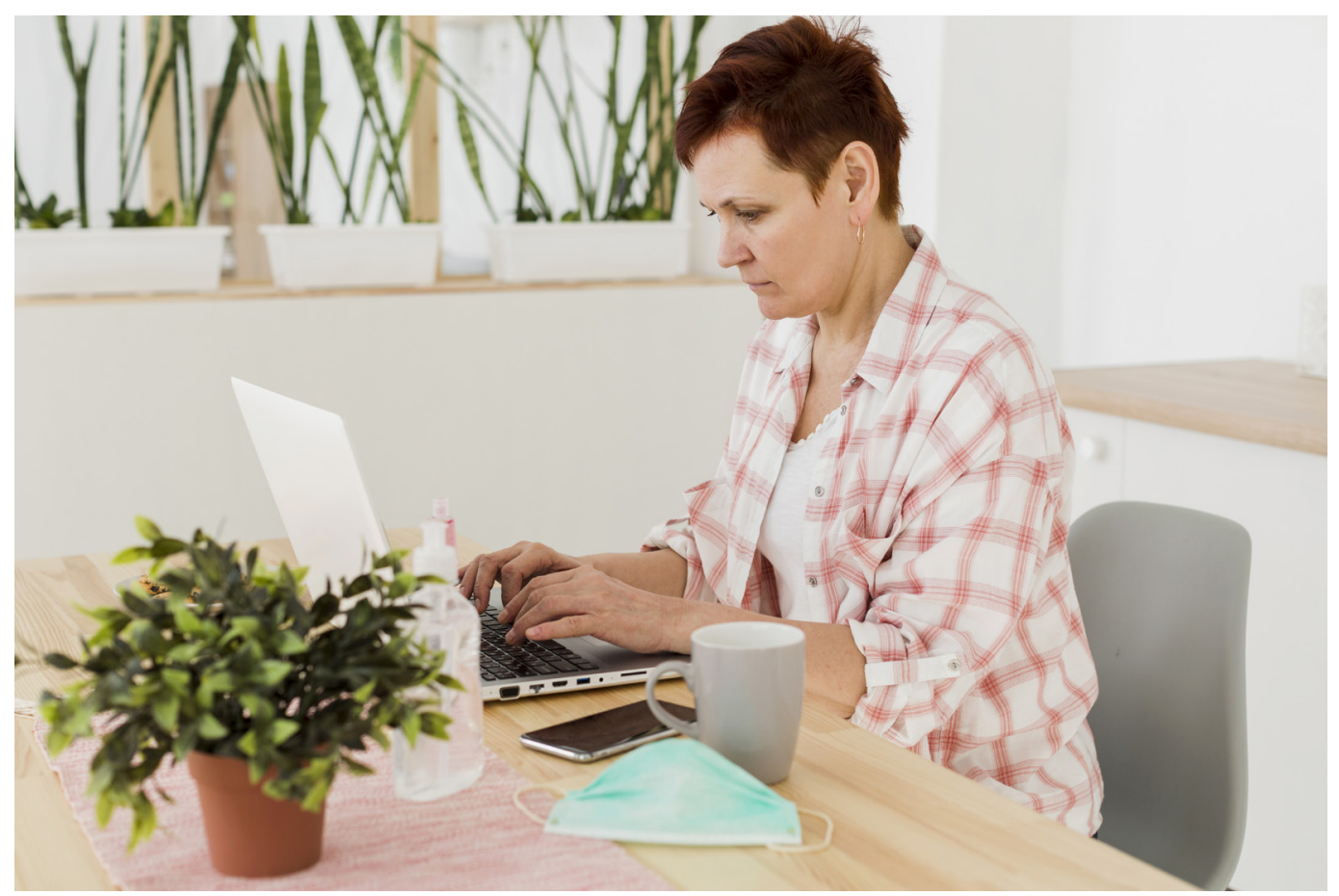


responsabilidad recaiga totalmente en ellos. Nosotros como docentes o instituciones educativas también participamos en dicho proceso y, por consiguiente, no podemos delegar la responsabilidad educativa únicamente a la familia.

\section{Instituciones educativas}

Las instituciones educativas son las encargadas de ofrecer un lugar donde se genera el proceso de enseñanza-aprendizaje y su tarea es brindar un espacio donde los estudiantes puedan recibir una educación de calidad, así como desarrollar un proceso de socialización sin impedimentos. Sin embargo, con la pandemia han surgido dificultades que no esperábamos en estos y otros aspectos.

La situación de las escuelas es muy interesante. Flores (2020) realizó una comparación entre dos institutos privados y pudo encontrar que una de esas dos escuelas se destacaba en conocimientos informáticos de una computadora, pero al mismo tiempo la otra institución se destacaba en aspectos como el uso de plataformas como Netflix. Esto no dista mucho de la realidad presente en todas las escuelas. Aunque tenemos problemáticas similares, cada escuela es distinta y si lo que buscamos es elevar el nivel académico, entonces, necesitamos atender las necesidades que afectan al proceso de enseñanza-aprendizaje de cada institución.

Las instituciones educativas siempre están en un proceso de transformación y, dado que debemos manejar la educación a distancia, éstas deben adaptarse a una nueva realidad, donde la meta principal es que la educación llegue a todos los estudiantes posibles. Con esto como punto de partida, podemos definir que el éxito en el rendimiento de los aprendices reside también en la escuela y no exclusivamente en el alumnado, por lo cual debemos de brindar un servicio que permita generar aprendizaje.

Domínguez Martínez (2010) expone que esta educación debe realizarse con una relación cordial entre docentes y padres de familia. Por ende, tanto los padres y profesores deben dar su apoyo continuo en el proceso educativo. En cuanto a este aspecto, Cabello (2016) relata que el marco de la participación de las familias parte de los mecanismos habilitados para ello, y es ahí donde podemos encontrar que se trata de un trabajo conjunto, que depende de todos y no de unos cuantos.

Para culminar, Pedro (2006) plantea que la participación de los padres de familia puede entorpecer la labor del maestro. Él menciona que existen padres sobreprotectores, que no dejan al docente trabajar o que quieren tener el control de la educación en sus manos. El docente es un agente que busca brindar educación y si algo lo detiene, entonces, no podrá proporcionarla. Con la tendencia al uso de nuevas tecnologías en esta realidad a distancia, los padres de familia deben también conocer cómo se usarán dichas herramientas, para que no surjan conflictos a futuro. Si los padres de familia le dan un uso incorrecto a la tecnología van a ocasionar más daño a los estudiantes. 


\section{Nuevas tecnologías}

Un detalle que llama la atención es que desde hace años las nuevas tecnologías han transformado el comportamiento de la sociedad y ésta se ha adaptado al mismo ritmo. En el caso de la educación, la tecnología ha aportado muchísimas herramientas que facilitan la tarea del proceso de enseñanza-aprendizaje. Como los aparatos tecnológicos como televisores, tabletas, teléfonos inteligentes y computadoras salen al mercado constantemente, éstos tienden a bajar su precio, lo cual permite que puedan ser obtenidos por una mayor cantidad de la población.

Solano (2013) afirma que las nuevas tecnologías ya son parte de las familias y que han cambiado la forma de comunicarnos. Prácticamente cualquier persona puede tener acceso a estos dispositivos, ya que su costo puede llegar a ser bajo. Maria (2010) señala, por su parte, que la educación ya se ha unido a los nuevos medios digitales y comenta que internet puede usarse para obtener nuevos conocimientos, así como facilitar nuestra vida ante los problemas cotidianos. A pesar de que éste es el objetivo de toda tecnología, se debe de tener una supervisión continua, porque las personas pueden desvirtuar su uso de forma muy fácil y volverla un distractor.

La educación a distancia ha existido desde hace bastante tiempo y ha cambiado en consecuencia. Esta modalidad, que se utiliza fundamentalmente como auxiliar en el proceso de enseñanza-aprendizaje, ha tomado fuerza en los últimos años, especialmente en los sectores de educación superior. Sin embargo, en 2020 y 2021, todas las escuelas en México se vieron forzadas a utilizar la educación a distancia, dados los riesgos sanitarios que conlleva tener clases presenciales en medio de una pandemia.

En el dominio de la tecnología, autores como Cabero-Almenara et al. (2020) afirman que los estudiantes están inmersos en redes sociales y que esto puede causar adicción. En su estudio, encontró que la adicción a estas plataformas puede ser explicada por la satisfacción/tolerancia, problemas, obsesión por estar informado y la necesidad de estar conectado.

Otros como García y Fernández (2016) destacan una fuerte presencia de dispositivos móviles en jóvenes y adolescentes. Si bien, las redes sociales pueden ser empleadas como distractores, también pueden utilizarse para comunicar, interactuar, y localizar y demandar información, e incluso para crear convivencia, diversión y nuevos lenguajes de comunicación.

\section{¿Cómo funciona la educación a distancia?}

Ko y Rossen (2001) definen la educación a distancia como un proceso que incluye cursos dictados a través de correo electrónico, video, conexión vía teléfono o por televisión satelital. Esta es una forma de aprendizaje que no implica la clase 
tradicional, en la cual los estudiantes y el instructor deben estar en el mismo lugar al mismo tiempo.

Kearsley (2002), por su parte, menciona las características de la educación en línea: colaboración, conectividad, que está centrada en el estudiante, que no tiene límites de lugar y tiempo, que puede ser explorada, tener conocimiento compartido, experiencia multisensorial y autenticidad. Todas estas características son sumamente importantes para poder llevar de forma fructífera este tipo de aprendizaje y sin ellas nos estancaríamos.

\section{¿Por qué avanzó tanto la educación a distancia?}

Lorenzo (2007) habla que este tipo de educación comenzó a ganar mucha fuerza debido a su apertura, flexibilidad, eficacia, economía, privacidad, individualización, socialización, interacción, innovación y acceso a la calidad. Como podemos notar, son bastantes los beneficios que ofrece, y bien usada tiene la posibilidad de elevar el nivel académico de quienes la reciben.

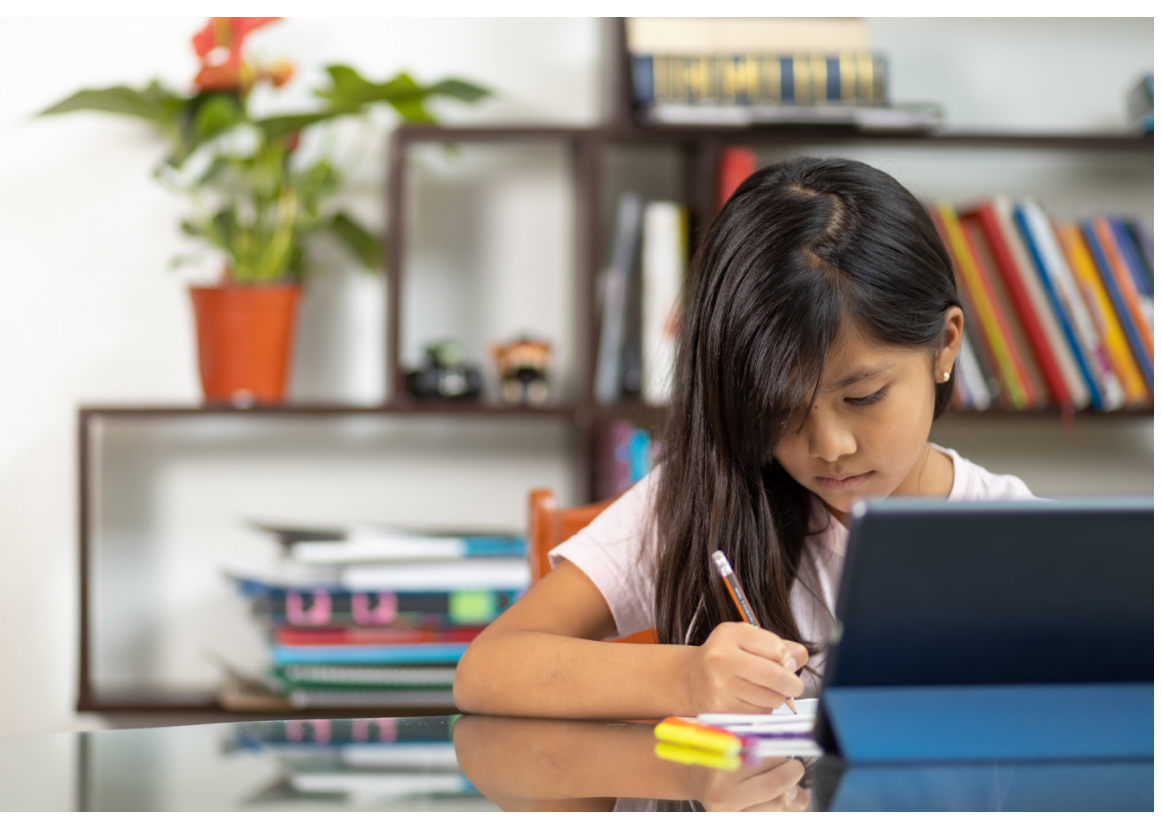

Estos aspectos, como el poder acceder a los temas de forma instantánea (con el beneficio de que cada día internet crece de forma inconmensurable), así como que la interacción sea más directa y que el retorno de comentarios quede evidenciado, pueden ser aprovechados de forma idónea, con mejores resultados a futuro. Si nosotros disponemos y utilizamos esto a nuestro favor los resultados serán superiores.

Aun así, no todo es benéfico en la educación a distancia. Borges en su artículo "La frustración del estudiante en línea. Causas y acciones preventivas" (2005) hace notar las frustraciones que tienen cada participante del proceso de enseñanza-aprendizaje. Para los estudiantes, éstas incluyen el no disponer de suficiente tiempo, las expectativas irreales, el no tener estrategias y destrezas adecuadas para la formación en línea, no participar en actividades colaborativas, desconocer los canales de ayuda y no tener en cuenta el coste económico añadido. Algunos de los alumnos, incluso, tienen miedo por el desconocimiento de lo que se avecina.

Del lado del docente, tenemos factores como el no dar respuesta o darla tardíamente en la retroalimentación, tener presencia nula en el aula, no ser claro con las indicaciones, no mostrar cercanía con los estudiantes y contribuir a la sobrecarga del aprendiz. Kearsley (2002) hace notar que la educación en línea no 
es para todos los docentes, ya que exige más tiempo, conocimientos avanzados en programas de computadora y, sobre todo, el que el educador disfrute hacer este tipo de modalidad. Si el docente no encuentra el gusto por ella, entonces, la experiencia que tanto él como el estudiante vivirán no será grata.

Obviamente, también debemos mencionar las preocupaciones de las instituciones educativas y entre ellas resaltan el ofrecer una ayuda técnica deficiente, no ofrecer la adecuada capacitación en línea al docente, no dar una formación preliminar al estudiante, brindar expectativas irreales a los aprendices, pérdida de matrícula y no tener organizado el curso. Todas estas preocupaciones se suman al impacto económico que han recibido instituciones privadas, donde parte de sus estudiantes han emigrado a escuelas públicas. En el mismo sentido, las escuelas públicas han recibido un aumento de estudiantes que necesitan ser educados a distancia, lo cual conlleva a nuevas problemáticas. Es fácil solicitar que todos los alumnos tengan acceso a un dispositivo para clases en línea, pero la realidad apunta a que no todos pueden permitirse un gasto como ese, por más económico que pueda llegar a ser el dispositivo.

Para terminar, Vivanco (2020) describe el papel de los padres de familia y el acompañamiento que han hecho en medio de la pandemia. Algunos son analfabetos tecnológicos y por ello no cuentan con las habilidades para brindar un apoyo ni supervisión. Asimismo, el tiempo que los padres de familia pueden dedicar al apoyo educativo es variable. Algunos han conservado su empleo mientras que otros lo han perdido y necesitan salir a buscar los recursos necesarios, lo que causa un abandono en la supervisión. En familias con un poder adquisitivo alto, algunas de estas dificultades pueden ser inexistentes, pero para las de escasos recursos incluso son un impedimento para obtener la educación necesaria. Una familia de bajos ingresos difícilmente podrá tener una computadora, internet, teléfono inteligente o incluso una televisión, por lo cual no podrían llevar a cabo la educación a distancia.

Estas problemáticas no son nuevas, han existido desde hace más de 15 años. Siguen con nosotros y no sólo han aumentado gracias a la pandemia, sino que también su impacto en el aspecto académico ha sido mayor.

\section{Áreas de oportunidad}

Churchill (2004) sugiere la creación de un comité de aprendizaje en línea, la recolección de datos para propósitos educativos, el uso de una plataforma para la institución y seguir creando cursos de capacitación en línea. Todo esto con el objetivo de eliminar los problemas más comunes que se enfrentan en la educación a distancia.

Otra área de oportunidad es que debemos generar capacitación docente, estudiantil, de padres de familia y, por supuesto, de directivos en el área de este tipo de enseñanza, ya que no es un sistema que todos dominemos y, cuando 
se es necesario aplicar de forma inmediata, se denotan las fallas. No hacerlo causará complicaciones que pueden llevar meses en ser resueltas, y eso es algo que no podemos permitirnos en estos momentos.

Basándonos en lo analizado por Belén y Lázaro (2017), dentro de las áreas de oportunidad para estudiantes y padres de familia encontramos: el acordar la dedicación necesaria para la educación a distancia, ajustar lo necesario para aumentar la cantidad y calidad del tiempo disponible, revisar el volumen de los trabajos exigidos, saber cómo solicitar ayuda, participar activamente, y conocer la forma en la cual se entregarán las evidencias. Como podemos observar, no son situaciones complejas de realizar y el que los capacitemos beneficiará en gran manera el proceso educativo.

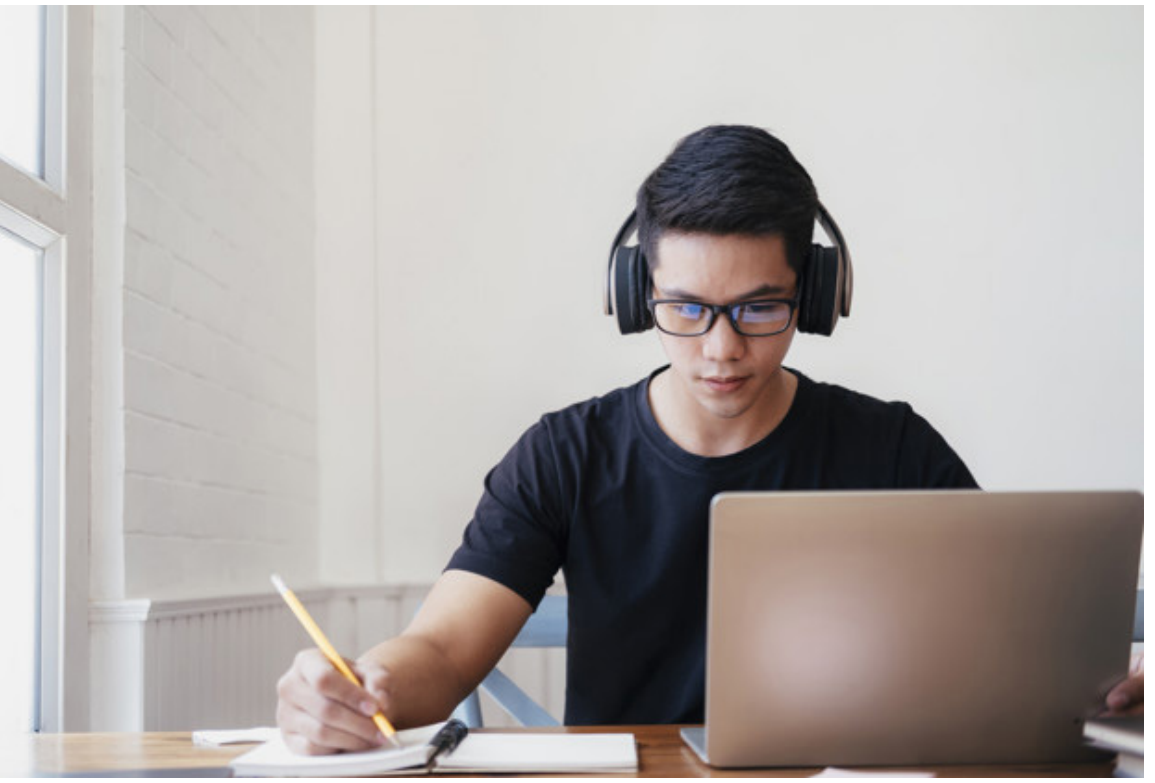
tomarnos tiempo en la clase para solucionar dudas emergentes, entre otras. Resulta necesario ser flexibles con los tiempos de entrega, sin embargo, eso no significa que los estudiantes los definan.

Para culminar, en el tema de áreas de oportunidad, encontramos que, para los directivos e instituciones educativas, se recomienda propiciar la colaboración entre docentes, ofrecer formación tanto para alumnos, padres de familia, docentes y directivos. Asimismo, el proporcionar ayuda técnica eficiente y asegurarse de que el material de aprendizaje sea adecuado servirá para poder tener éxito y, por ende, un servicio de calidad.

\section{Conclusiones}

Con la llegada del nuevo ciclo escolar 2021-2022, en México y muchos otros países, existe preocupación sobre la eficacia de la modalidad a distancia. Con 
plataformas como Google Classroom, Microsoft Teams, Webex, Zoom o los canales de televisión que brindaron su apoyo para impartir clases, el ofrecer un servicio de enseñanza a distancia no es complejo, lo difícil es asegurarse de poder dar una experiencia de calidad al educando, para que pueda desarrollar su proceso de enseñanza-aprendizaje.

Si queremos elevar el nivel educativo y desarrollar el proceso de enseñanzaaprendizaje, debemos llevar a cabo acciones como trabajar en conjunto. No obstante, dichas acciones deben ser constantes y no efímeras.

Nadie esperaba esta pandemia y todos hemos sido afectados de alguna forma u otra. Saldremos de esta situación, pero la clave reside en lo que estamos haciendo en este momento para superarla. Por ello, es vital que ofrezcamos lo mejor que podamos en la modalidad de educación a distancia.

\section{Referencias}

* Andrés Cabello, S. y Giró Miranda, J. (2016). La participación de las familias en la escuela: una cuestión compleja. Revista De Evaluación De Programas y Políticas Públicas, 1(7), 28-47. https://doi.org/10.5944/reppp.7.2016.16302

* Balarin, M. y Cueto, S. (2008). La calidad de la participación de los padres de familia y el rendimiento estudiantil en las escuelas públicas peruanas. Grade; Niños del Milenio.

* Rodríguez Fernández, S. y Romero Lozano, M. (2015, mayo-agosto). La función tutorial en Educación Infantil y Primaria: desempeño profesional del profesorado. Revista Electrónica Interuniversitaria de Formación del Profesorado, 18(2),43-56. https://www.redalyc.org/articulo.oa?id=217036214005

* Borges, F. (2005). La frustración del estudiante en línea. Causas y acciones preventivas. Digithum, (7), 0. https://www.redalyc.org/articulo.oa?id=55000706

* Cabero-Almenara, J., Pérez-Díez de los Ríos, J. L. y Valencia-Ortiz, R. (2020). Escala para medir la adicción de estudiantes a las redes sociales. Convergencia, 27, e11834. https://doi.org/10.29101/crcs.v27i0.11834

* Churchill, A. (2004). Ensuring quality in online higher education courses. University of Massachusetts.

* Domínguez Martínez, S. (2010, mayo). La Educación, cosa de dos: La escuela y la familia. Temas para la educación. Revista digital para profesionales de la enseñanza, (8). https://www.feandalucia.ccoo.es/docu/p5sd7214.pdf

* Flores, E. (2020). Competencias digitales en educación secundaria, una comparación entre dos escuelas privadas de la ciudad de Durango. Redie.

* García, M. y Fernández, C. [Coords.] (2016). Si lo vives, lo compartes. Cómo se comunican los jóvenes en un mundo digital. Fundación Telefónica-Ariel.

* Kearsley, G. (2002, enero-febrero). Is online learning for everybody? Educational Technology, 42(1), 41-44. https://www.jstor.org/stable/44428721 
* Ko, S. y Rossen, S. (2001). Teaching online: A practical guide. Houghton Mifflin.

- Lorenzo, G. (2007). Por quéva ganando la educación a distancia. Editorial del Bened.

* Maria, R. L. (2010, enero-abril). El uso de las nuevas tecnologías de la información y la comunicación (NTICS). Revista Electrónica "Actualidades", 10(1), 1-13. https://www. redalyc.org/articulo.oa?id=44713068018

* Ramírez Ruiz, M. y de Andrade, D. (2005). La familia y los factores de riesgo relacionados con el consumo de alcohol y tabaco en los niños y adolescentes (Guayaquil-Ecuador). Revista Latino-Americana de Enfermagem, 13(1), 813-818. https://www.redalyc.org/articulo.oa?id=281421851008

* Solano, A. y Viñaras, M. (2013). Las nuevas tecnologías en la familia y la educación: retos y riesgos de una realidad inevitable. Ediciones CEU.

* Tobón, S., Carretero Díaz, M. Á, García Fraile, J. A. y Rial Sánchez, A. (2006). Competencias, calidad y educación superior. Cooperativa Editorial Magisterio.

* Vivanco-Saraguro, A. (2020). Teleducación en tiempos de covid-19: brechas de desigualdad. CienciAméRica, 9(2), 166-175. https://doi.org/10.33210/ca.v9i2.307

\section{Cómo CITAR ESTE ARTículo}

* Haro Piña, Alexis. (2021, noviembre-diciembre). Buscar el mejoramiento docente en la educación a distancia. Revista Digital Universitaria (RDU), 22(6). http://doi. org/10.22201/cuaieed.16076079e.2021.22.6.8 